\title{
Bedside Sublingual Video Imaging of Microcirculation in Assessing Bacterial Infection in Cirrhosis
}

\author{
Muhammad Y. Sheikh · Usman Javed · Jasjit Singh • \\ Jayanta Choudhury · Omer Deen · Kulraj Dhah · \\ Michael W. Peterson
}

Received: 26 September 2008 / Accepted: 8 December 2008/Published online: 1 January 2009

(C) The Author(s) 2008. This article is published with open access at Springerlink.com

\begin{abstract}
Bacterial infections are common in cirrhosis and can lead to life-threatening complications. Sidestream dark-field (SDF) imaging has recently emerged as a noninvasive tool for capturing real-time video images of sublingual microcirculation in critically ill patients with sepsis. The objective of this study was to assess the utility of SDF in determining underlying infection in patients with cirrhosis. Sublingual microcirculation was compared among patients with compensated cirrhosis (Group A, $n=13$ ), cirrhosis without sepsis (Group B, $n=18$ ), cirrhosis with sepsis (Group C, $n=14$ ), and sepsis only (Group $\mathrm{D}, n=10$ ). The blood flow was semi-quantitatively evaluated in four equal quadrants in small (10$25 \mathrm{~mm}$ ); medium (26-50 mm); and large (51-100 mm) sublingual capillaries. The blood flow was described as no flow (0), intermittent flow (1), sluggish flow (2), and continuous flow (3). The overall flow score or microvascular flow index (MFI) was measured for quantitative assessment of microcirculation and predicting power for concurrent infection in cirrhosis. Marked impairment was observed at all levels of microvasculature in Groups B and $\mathrm{C}$ when compared with Group A. This effect was restricted to small
\end{abstract}

M. Y. Sheikh $(\bowtie) \cdot$ J. Choudhury · O. Deen · K. Dhah Division of Gastroenterology and Hepatology, University of California San Francisco-Fresno Medical Education Program, Community Regional Medical Center, 2823 Fresno Street, 1st Floor, Endoscopy Suite, Fresno, CA 93721, USA

e-mail: msheikh@ fresno.ucsf.edu

U. Javed

Division of Cardiology, University of California San FranciscoFresno Medical Education Program, Fresno, CA, USA

J. Singh · M. W. Peterson

Department of Internal Medicine, University of California San Francisco-Fresno Medical Education Program, Fresno, CA, USA vessels only when Group B was compared with Group C. MFI $<1.5$ was found to have highest sensitivity $(100 \%)$ and specificity $(100 \%)$ for infection in decompensated cirrhosis. SDF imaging of sublingual microcirculation can be a useful bedside diagnostic tool to assess bacterial infection in cirrhosis.

Keywords Cirrhosis - Spontaneous bacterial peritonitis . Microcirculation · Sepsis · Sidestream dark-field imaging · Microvascular flow index

\section{Introduction}

Bacterial infections are major causes of morbidity and mortality in cirrhosis $[1,2]$. Cirrhosis is associated with alterations in gut motility, bacterial flora, and intestinal permeability, thus favoring bacterial translocation [3]. As a consequence, endotoxemia has been noted in cirrhosis without infection [4]. More recently, high plasma levels of TNF- $\alpha$, IL1- $\beta$, IL6, and soluble adhesion molecules have been demonstrated in decompensated cirrhosis, further supporting the presence of an on-going inflammatory response in this patient population [5-8].

Microcirculatory function is the main prerequisite for adequate tissue oxygenation during infection or sepsis [9, 10]. As microcirculation is the primary site of oxygen and nutrient exchange, the persistence of microcirculatory dysfunction can lead to tissue hypoxia, organ failure, and death. Thus, monitoring microcirculation is regarded as a valuable tool in assessing the severity of the disease and predicting its outcome [11]. This phenomenon has not yet been studied in patients with cirrhosis.

Recent developments in new medical imaging techniques have helped to discover microcirculation as a key 
player in sepsis [12]. Several methods [12-19] have been used to monitor microcirculatory function during circulatory failure in surgery and intensive care. Orthogonal polarization spectral (OPS) imaging has enabled the first direct observation of the microcirculation of human internal organs $[18,19]$. More recently, sidestream dark-field (SDF) imaging has emerged as a next-generation noninvasive bedside tool that provides high contrast and realtime video images of the microcirculation in critically ill patients with sepsis $[9,12,20,21]$.

This prospective study was designed to assess changes in the sublingual microcirculation with SDF video imaging in decompensated cirrhosis with and without infection. The primary goal was to determine whether sublingual microcirculatory changes could be a diagnostic tool to identify infection in cirrhosis.

\section{Methods}

Patients

We enrolled 55 consecutive patients between 18 and 65 years with cirrhosis due to common etiologies over six months at our institution. Patients were categorized into four groups as follows:

A. 14 patients with compensated cirrhosis without infection

B. 18 patients with decompensated cirrhosis without clinical infection or sepsis

C. 13 patients with decompensated cirrhosis and infection or sepsis

D. 10 patients with sepsis but without cirrhosis.

All subjects in Groups C and D were hospitalized patients whereas subjects in Groups A and B were all outpatients. None of the Group A and Group B patients was on antibiotic therapy at the time of enrollment or had clinical evidence of any infection. In Groups $\mathrm{C}$ and D, video imaging of microcirculation was accomplished within $48 \mathrm{~h}$ of admission and initiation of antibiotic therapy. Baseline characteristics for each subgroup were assessed, including platelet counts, and Child-TurcottePugh (CTP) and model of end-stage liver disease (MELD) scores (Table 1). Sepsis was identified on the basis of American College of Chest Physicians/Society of Critical Care Medicine consensus conference criteria [22]. Patients with alcoholic hepatitis, pregnancy, HIV, hypotension (systolic blood pressure $<90 \mathrm{mmHg}$ ), DIC, acute myocardial infarction, congestive heart failure, chronic renal failure, malignancies, and hypercoagulable states were excluded.
Table 1 Baseline characteristics of patients

\begin{tabular}{|c|c|c|c|}
\hline & $\begin{array}{l}\text { Group A } \\
N=14\end{array}$ & $\begin{array}{l}\text { Group B } \\
N=18\end{array}$ & $\begin{array}{l}\text { Group C } \\
N=13\end{array}$ \\
\hline \multicolumn{4}{|l|}{ Age (years) } \\
\hline Mean & 40 & 55 & 54 \\
\hline \multicolumn{4}{|l|}{ Gender } \\
\hline Male & 9 & 11 & 7 \\
\hline Female & 5 & 7 & 6 \\
\hline \multicolumn{4}{|l|}{ Race } \\
\hline Hispanic & 13 & 17 & 13 \\
\hline White & 1 & - & - \\
\hline Other & - & 1 & - \\
\hline \multicolumn{4}{|l|}{ MELD score } \\
\hline Range & $7-10$ & $8-16$ & $10-29$ \\
\hline Mean & 8 & 12 & 20 \\
\hline \multicolumn{4}{|l|}{ CTP score } \\
\hline Range & $5-7$ & $7-11$ & $9-12$ \\
\hline Mean & 6 & 8 & 11 \\
\hline \multicolumn{4}{|c|}{ Platelets count } \\
\hline Range & 100-208 & $50-170$ & $41-89$ \\
\hline Mean & 150 & 110 & 65 \\
\hline \multicolumn{4}{|l|}{ Etiology } \\
\hline $\mathrm{HCV}$ & 3 & 5 & 2 \\
\hline Alcohol & 3 & 8 & 3 \\
\hline Both & 3 & 4 & 4 \\
\hline Other & 5 & 1 & 4 \\
\hline
\end{tabular}

Group A, compensated cirrhosis; Group B, decompensated cirrhosis without infection/sepsis; Group C, decompensated cirrhosis with infection/sepsis

\section{Methodology}

All evaluations of microvascular flow were performed by the same two investigators (OD and KD) as described by De Backer et al. [20] and Boerma et al. [21]. In Groups C and $\mathrm{D}$, microvascular flow was evaluated within $48 \mathrm{~h}$ of admission. A small probe (MicroScan; MicroVisionMedical) was placed under the tongue and streaming images of the microcirculation were obtained and stored on digital videotape (Sony Video Walkman GV-D 1000E). As heterogeneity of flow has been postulated to be an important characteristic of microvascular alterations during sepsis [23], multiple video clips were obtained from three different sublingual locations. At least three good-quality sequences of $20 \mathrm{~s}$ were taken from each site of interest. Of each individual video clip, the best quality segment was selected for semi-quantitative analysis of microvascular flow. The selection of video segments was dependent on image quality (to avoid saliva, air bubbles, and pressure artifacts). 
Imaging and Analysis Procedure

Each image was divided into four equal quadrants (I, II, II, and IV) (Fig. 1). Quantitative flow was scored per quadrant, for each cohort of vessel diameter. Microvascular flow was quantified by observation as no flow $(0$, no flow for $20 \mathrm{~s}$ ), intermittent flow (1, greater than $10 \mathrm{~s}$ with no flow), sluggish flow (2, less than $10 \mathrm{~s}$ with no flow), and continuous flow (3, continuous flow for 20 s). If a quadrant contained different types of vessel with different diameters (e.g. the sublingual vascular bed), these quantifications of flow were made according to the cohort of vessel diameter: small $(10-25 \mu \mathrm{m})$; medium $(26-50 \mu \mathrm{m})$; and large, $(51-$ $100 \mu \mathrm{m})$.

The overall score, called the microvascular flow index (MFI), was obtained as the sum of each quadrant score divided by the number of quadrants in which the vessel type was visible. MFI was quantified by two independent observers (UJ and JS), who were unaware of patient information. When there was a disagreement between these two observers, the same third observer (MYS) reviewed the images for quantification of MFI. Inter-observer and intraobserver disagreement was 12 and 10\%, respectively. For each subject, MFI was calculated on the basis of the four quadrants of three video clips from three areas of interest.

\section{Statistical Analysis}

MFI was measured for small, medium, and large capillaries. The mean for each group was used to perform a subgroup analysis. The data from the four groups were tested using ANOVA and, finally, all four groups were compared by use of Bonferroni corrections (Tables 2 and 3).

\section{Results}

Mean patient age in compensated and decompensated cirrhosis was 40 and 55 years, respectively. Most patients (98\%) were Latinos and the most common causes of cirrhosis were alcohol and chronic hepatitis $\mathrm{C}$. The underlying infections in Group C were spontaneous bacterial peritonitis (five patients), cellulitis (three patients), communityacquired pneumonia (three patients), acute osteomyelitis (one patient), and salmonella bacteremia (one patient). The mean MELD scores were 8, 12, and 20 for Groups A, B, and $\mathrm{C}$ respectively. An increase in MELD and CTP scores and a decline in platelet counts from Groups A to C reflected the severity of liver disease (Table 1 ). The values for Groups A (2.5 $\pm 0.2 \mathrm{SD}$, CI 2.3-2.6), B (1.5 $\pm 0.3 \mathrm{SD}$, CI 1.3-1.8), and C (1.2 $\pm 0.2 \mathrm{SD}, \mathrm{CI} 1.0-1.4$.$) are listed in$ Table 2. Only Group A (compensated cirrhosis) differed from the other groups (Table 3). Microcirculation impairment was most noticeable in the small capillaries, irrespective of study group. Microcirculatory flow was significantly impaired in all capillary beds in Group B (decompensated cirrhosis) $(P<0.001)$ and $\mathrm{C}$ (decompensated cirrhosis with infection) $(P<0.001)$ when compared with Group A. Groups B and C differed only at the small capillary level $(P<0.033)$. Neither Group B nor Group C
Fig. 1 Sidestream dark-field video imaging demonstrating blood flow in a cirrhosis patient with infection. The blood is measured in various sized capillaries in four quadrants of the video clip
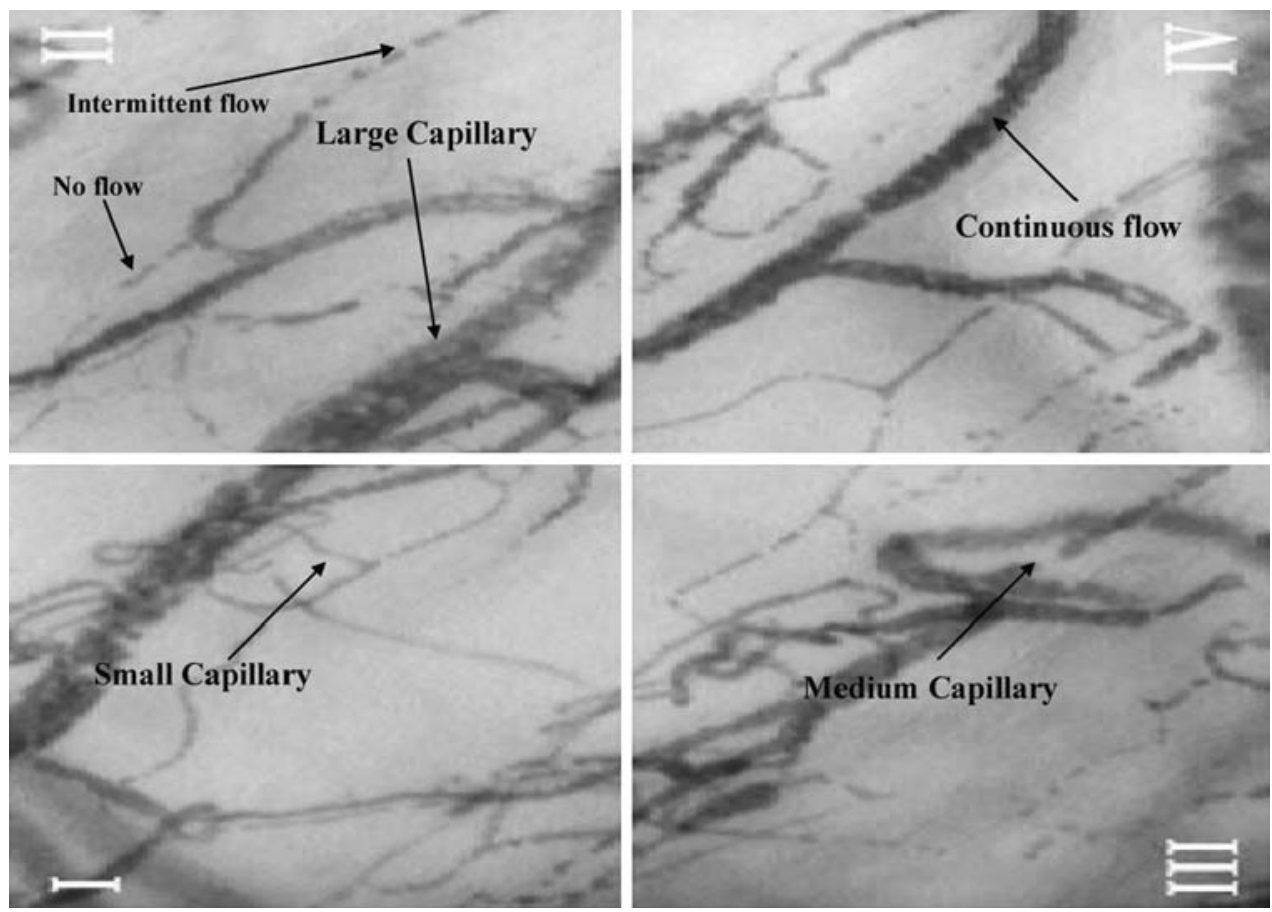
Table 2 Microvascular flow index (MFI) in the four study groups in relation to vessel size

\begin{tabular}{llll}
\hline Vessel & Group & MFI (mean \pm SD) & $95 \%$ CI \\
\hline Small & A & $2.40 \pm 0.46$ & $2.07-2.73$ \\
& B & $1.40 \pm 0.52$ & $1.03-1.77$ \\
& C & $0.80 \pm 0.48$ & $0.45-1.15$ \\
Medium & D & $1.15 \pm 0.34$ & $0.45-1.39$ \\
& A & $2.35 \pm 0.42$ & $2.05-2.64$ \\
& B & $1.40 \pm 0.32$ & $1.17-0.62$ \\
& C & $1.25 \pm 0.48$ & $0.90-0.59$ \\
Large & D & $1.55 \pm 0.28$ & $1.34-0.75$ \\
& $\mathrm{~A}$ & $2.75 \pm 0.35$ & $2.50-3.00$ \\
& $\mathrm{~B}$ & $1.90 \pm 0.77$ & $1.35-0.45$ \\
& $\mathrm{C}$ & $1.65 \pm 0.47$ & $1.31-1.99$ \\
Mean $^{\mathrm{a}}$ & $\mathrm{D}$ & $2.10 \pm .52$ & $1.73-0.47$ \\
& $\mathrm{~A}$ & $2.50 \pm 0.21$ & $2.35-2.64$ \\
& $\mathrm{~B}$ & $1.56 \pm 0.35$ & $1.31-1.81$ \\
& $\mathrm{C}$ & $1.23 \pm 0.24$ & $1.06-1.40$ \\
& $\mathrm{D}$ & $1.60 \pm 0.32$ & $1.37-1.82$ \\
\hline
\end{tabular}

Group A, compensated cirrhosis; Group B, decompensated cirrhosis without infection/sepsis; Group C, decompensated cirrhosis with infection/sepsis; Group D, sepsis without cirrhosis

${ }^{a}$ Mean of all vessel types in an individual study group

differed from Group D (sepsis patients) in any capillary bed. Mean microvascular flow index (MFI) between 1.0 and 1.5 best predicted underlying infection in cirrhosis with sensitivity and specificity approaching 100\% (Table 4).

\section{Discussion}

We used SDF video imaging of microcirculation for the first time in cirrhosis to assess infections. Our study demonstrated that the sublingual microcirculation in decompensated cirrhosis mimics changes seen in sepsis but with less intensity. These findings suggest that similar mechanisms might exist in decompensated cirrhosis without clinical infection. Alterations in gut bacterial flora, bacterial translocation [4] deficient phagocytosis [23], lower complement levels [24], poor opsonization, and impaired serum bacteriocidal activity [25] all contribute to increased susceptibility to infection in cirrhosis. High plasma levels of tumor necrosis factor $\alpha$ (TNF- $\alpha$ ) interleukin (IL)-1, IL-6, and soluble adhesion molecules have recently been demonstrated in decompensated cirrhosis $[26,27]$. In addition, patients with cirrhosis show high plasma concentrations of endothelin (ET), and the levels correlate with disease severity and ascites [27]. Therefore, patients with decompensated cirrhosis are in the state of
Table 3 Comparison of microvascular flow index (MFI) among various study groups

\begin{tabular}{|c|c|c|c|}
\hline Vessel type & Group & Mean MFI difference & $P$ \\
\hline \multirow[t]{6}{*}{ Small } & A vs. B & 1.00 & $<0.0001$ \\
\hline & A vs. C & 1.60 & $<0.0001$ \\
\hline & A vs. D & 1.25 & $<0.0001$ \\
\hline & $\mathrm{B}$ vs. $\mathrm{C}$ & 0.60 & .033 \\
\hline & B vs. D & 0.25 & 1.000 \\
\hline & $\mathrm{C}$ vs. $\mathrm{D}$ & 0.35 & .561 \\
\hline \multirow[t]{6}{*}{ Medium } & A vs. B & 0.95 & $<0.0001$ \\
\hline & A vs. C & 1.10 & $<0.0001$ \\
\hline & A vs. D & 0.80 & $<0.0001$ \\
\hline & B vs. $\mathrm{C}$ & 0.15 & 1.000 \\
\hline & B vs. D & 0.15 & 1.000 \\
\hline & $\mathrm{C}$ vs. $\mathrm{D}$ & 0.30 & .529 \\
\hline \multirow[t]{6}{*}{ Large } & A vs. B & 0.85 & 0.009 \\
\hline & A vs. C & 1.10 & $<0.0001$ \\
\hline & A vs. D & 0.65 & .074 \\
\hline & B vs. C & 0.25 & 1.000 \\
\hline & B vs. D & 0.20 & 1.000 \\
\hline & $\mathrm{C}$ vs. $\mathrm{D}$ & 0.45 & .458 \\
\hline \multirow[t]{6}{*}{ Mean $^{a}$} & A vs. B & 0.93 & $<0.0001$ \\
\hline & A vs. C & 1.27 & $<0.0001$ \\
\hline & A vs. D & 0.90 & $<0.0001$ \\
\hline & B vs. C & 0.33 & .077 \\
\hline & B vs. D & 0.03 & 1.000 \\
\hline & C vs. D & 0.37 & .501 \\
\hline
\end{tabular}

Group A, compensated cirrhosis; Group B, decompensated cirrhosis without infection/sepsis; Group C, decompensated cirrhosis with infection/sepsis; Group D, sepsis without cirrhosis

${ }^{a}$ Mean of all vessel types in an individual study group

Table 4 Discriminatory power of microvascular flow index (MFI) for infection/sepsis at various cut-off levels

\begin{tabular}{lccrr}
\hline MFI cut off & Sensitivity & Specificity & PPV & NPV \\
\hline$<1.0$ & 90 & 100 & 100 & 91 \\
$<1.5$ & 100 & 100 & 100 & 100 \\
$<2.0$ & 100 & 50 & 60 & 100 \\
\hline
\end{tabular}

endotoxemia without overt infection [28]. This endotoxemia, with cytokine activation, ET release, and other hormonal changes, could contribute significantly to vasoconstriction in the setting of arteriovenous shunting seen in decompensated cirrhosis. In clinical bacterial infection, larger concentrations of endotoxins, cytokines, ET-1, catecholamines, and angiotensin II are produced. The resulting net vasoconstriction leads to hypoxia, which is also a driving force for ET-1 release. It is very likely that overall ET receptor stimulation depends on the relative balance between the competing vasodilator and 
vasoconstrictor forces $[29,30]$. The balance is ultimately tipped in the favor of vasoconstrictors in advanced cirrhosis and associated infection.

Blood flow through capillaries is quite different from that through arterioles and venules. Exchange of metabolites and transport through the vessel wall is only possible in the capillaries, because only here is the blood flow sufficiently reduced (about $0.3 \mathrm{~mm} / \mathrm{s}$ ) and the vessel wall sufficiently thin. The arterioles are of larger diameter $(\leq 50 \mu \mathrm{m})$ and are responsible for vascular resistance. Arteriovenous shunts formed in advanced cirrhosis cause some diversion of blood flow away from the true capillaries, thus affecting microcirculation [31, 32]. In addition, impaired red cell deformability has been found in patients with cirrhosis and sepsis. This increased rigidity of the red cells could, in part, explain the AV shunting and decreased microcirculatory flow seen in both cirrhosis and sepsis [33]. We believe that the changes we found in medium and small capillaries in decompensated cirrhosis could also be the result of $\mathrm{AV}$ shunting and impaired red cell deformability.

When compared with the Group B patients, Group C patients had higher MELD and CTP scores and evidence of clinical infection. For these patients a profound impact on their microcirculation was observed at the smaller capillary levels ( $\Delta$ MFI $0.6, P=0.03$.). Hardly any flow was noticed in these smaller capillaries. Therefore, MFI $<1.5$ represented the most sensitive and specific tool for detecting infection (Table 4). These vascular changes could represent the cumulative effects of increasing cytokines, ET, and other vasoconstrictors in the presence of worsening hemodynamics of advanced cirrhosis. With infection, we expect a further rise in these cytokines that can aggravate vascular resistance and consequent decreased perfusion. The cytokines may also decrease nitric oxide release and endothelial-dependent relaxation noted in smaller vessels [34]. Besides vasoconstriction, the accumulation and adherence of leukocytes to the vascular wall seen in sepsis [35] may worsen microcirculation. Tissue hypoxia can further induce an inflammatory response within the vessel wall, and the recruited circulating progenitor cells could contribute significantly to the structural remodeling and persistent vasoconstriction [36]. In addition to CTP and MELD scores, hemodynamic changes in patients with cirrhosis correlate with their prognosis [37]. Our study supports a good correlation between these scores and microcirculatory changes.

Despite many interesting observations made through a selected group of patients, our study has many limitations. First, the number of subjects in the study groups (B and C) was relatively small. Second, Group $\mathrm{C}$ had heterogeneous infections. Third, microcirculation was assessed 24-48 h after initiation of antibiotic therapy that might have improved patients' clinical sepsis in Group C. The latter might have affected our findings, which would have been significantly worse if microcirculation had been assessed at the time of patients' initial presentation. Fourth, invasive hemodynamic data and other hormonal factors for these patients were not assessed and in future, larger studies may have to address the need for such monitoring in the context of this technique.

As sepsis in cirrhosis is associated with complications such as encephalopathy, gastrointestinal bleed, and renal failure, the observations noted in our study raise several interesting questions. Does hypoxia induced by impaired systemic circulation aggravate hepatic encephalopathy seen in patients with worsening liver disease or infection? Do worsening microcirculatory changes predict advancing cirrhosis, portal hypertension, or hepatorenal syndrome? Are systemic microcirculatory changes reversible with antibiotic therapy with or without albumin infusion? Prospective studies are currently needed to establish such relationships in a larger patient population.

In conclusion, our study has demonstrated that the microcirculation is affected in both cirrhosis and in sepsis without liver disease. These microcirculatory changes in decompensated cirrhosis with overt infection are profoundly impaired and can be detected noninvasively by assessing sublingual microcirculation at bedside. This method of monitoring microcirculation at the bedside in cirrhotics has great potential in overall management of this complex disorder. Early non-invasive monitoring of microcirculation in infections such as spontaneous bacterial peritonitis can help physicians to decide the timing of initiation of antibiotic therapy and thus prevent lifethreatening complications of cirrhosis. Larger studies are needed to further assess the validity of sublingual imaging of microcirculation in cirrhosis with infection before and after therapeutic intervention.

Open Access This article is distributed under the terms of the Creative Commons Attribution Noncommercial License which permits any noncommercial use, distribution, and reproduction in any medium, provided the original author(s) and source are credited.

\section{References}

1. Guarner C, Runyon BA. Macrophages function in cirrhosis and the risk of bacterial infection. Hepatology. 1995;22:367-369.

2. Wong F, Bernardi M, Balk R, et al. Sepsis in cirrhosis: report on the 7th meeting of the International Ascites Club. Gut. 2005;54:718-725. doi:10.1136/gut.2004.038679.

3. Runyon BA. Early events in spontaneous bacterial peritonitis. Gut. 2004;53:782-784. doi:10.1136/gut.2003.035311.

4. Bauer TM, Schwacha H, Steinbruckner B, et al. Small intestinal bacterial overgrowth in human cirrhosis is associated with systemic endotoxemia. Am J Gastroenterol. 2002;97:2364-2370. doi:10.1111/j.1572-0241.2002.05791.x.

5. Such J, Hillebrand DJ, Guarner C, et al. Tumor necrosis factoralpha, interleukin-6, and nitric oxide in sterile ascetic fluid and 
serum from patients with cirrhosis who subsequently develop ascetic fluid infection. Dig Dis Sci. 2001;46:2360-2366. doi: 10.1023/A:1012342929326.

6. Tilg H, Wilmer A, Vogel W, et al. Serum levels of cytokines in chronic liver diseases. Gastroenterology. 1992;103:264-274.

7. Girón-González JA, Martínez-Sierra C, Rodríguez-Ramos C, et al. Implication of inflammation-related cytokines in the natural history of liver cirrhosis. Liver Int. 2004;24:437-445. doi: 10.1111/j.1478-3231.2004.0951.x.

8. Genesca J, Gonzalez A, Segura R, et al. Interleukin-6, nitric oxide, and the clinical and hemodynamic alterations of patients with liver cirrhosis. Am J Gastroenterol. 1999;94:169-177. doi: 10.1111/j.1572-0241.1999.00790.x.

9. Ince C. The microcirculation is the motor of sepsis. Crit Care. 2005;9:S13-S19. doi:10.1186/cc3753.

10. Spronk PE, Zandstra DF, Ince C. Bench-to-bedside review: sepsis is a disease of the microcirculation. Crit Care. 2004;8:462-468. doi:10.1186/cc2894.

11. Sakr Y, Dubois MJ, De Backer D, Creteur J, Vincent JL. Persistent microcirculatory alterations are associated with organ failure and death in patients with septic shock. Crit Care Med. 2004;32:18251831. doi:10.1097/01.CCM.0000138558.16257.3F.

12. Verdant $\mathrm{C}$, De Backer D. How monitoring of the microcirculation may help us at the bedside. Curr Opin Crit Care. 2005;11:240244. doi:10.1097/01.ccx.0000158849.94225.11.

13. Siegemund $\mathbf{M}$, van Bommel J, Ince C. Assessment of regional tissue oxygenation. Intensive Care Med. 1999;25:1044-1060. doi:10.1007/s001340051011.

14. Creteur J, De Backer D, Sakr Y, Koch M, Vincent JL. Sublingual capnometry tracks microcirculatory changes in septic patients. Intensive Care Med. 2006;32:516-523. doi:10.1007/s00134-0060070-4.

15. Guzman JA, Dikin MS, Kruse JA. Lingual, splanchnic, and systemic hemodynamic and carbon dioxide tension changes during endotoxic shock and resuscitation. J Appl Physiol. 2005;98:108-113. doi:10.1152/japplphysiol.00243.2004.

16. Weil MH, Nakagawa Y, Tang W, et al. Sublingual capnometry: a new noninvasive measurement for diagnosis and quantitation of severity of circulatory shock. Crit Care Med. 1999;27:12251229. doi:10.1097/00003246-199907000-00001.

17. De Backer D, Hollenberg S, Boerma C, et al. How to evaluate the microcirculation: report of a round table conference. Crit Care. 2007;11:R101. doi:10.1186/cc6118.

18. Groner W, Winkelman JW, Harris AG, et al. Orthogonal polarization spectral imaging: a new method for study of the microcirculation. Nat Med. 1999;5:1209-1212. doi:10.1038/13529.

19. Mathura KR, Vollebregt KC, Boer K, De Graaff JC, Ubbink DT, Ince C. Comparison of OPS imaging and conventional capillary microscopy to study the human microcirculation. J Appl Physiol. 2001;91:74-78.

20. De Backer D, Creteur j, Vincent JL, Dubois MJ, Preiser JC. Microvascular blood flow is altered in patients with sepsis. Am J Respir Crit Care Med. 2002;166:98-104. doi:10.1164/rccm. 200109-016OC.

21. Boerma EC, Mathura KR. Quantifying bedside-derived imaging of microcirculatory abnormalities in septic patients: a prospective validation study. Crit Care. 2005;9:R601-R606. doi:10.1186/ cc3809.

22. Cohen J, Guyatt G, Bernard GR, et al. New strategies for clinical trials in patients with sepsis and septic shock. Crit Care Med. 2001;29:880-886. doi:10.1097/00003246-200104000-00039.

23. Panasiuk A, Wysocka J, Maciorkowska E, et al. Phagocytic and oxidative burst activity of neutrophils in the end stage of liver cirrhosis. World J Gastroenterol. 2005;11:7661-7665.

24. Bird G, Senaldi G, Panos M, et al. Activation of the classical complement pathway in spontaneous bacterial peritonitis. Gut. 1992;33:307-311. doi:10.1136/gut.33.3.307.

25. Runyon BA. Patients with deficient ascitic fluid opsonic activity are predisposed to spontaneous bacterial peritonitis. Hepatology. 1988;8:535-632. doi:10.1002/hep.1840080332.

26. Byl B, Roucloux I, Crusiaux A, Dupont E, Devière J. Tumor necrosis factor alpha and interleukin 6 plasma levels in infected cirrhotic patients. Gastroenterology. 1993;104:1492-1497.

27. Alam I, Bass NM, Bacchetti P, Gee L, Rockey DC. Hepatic tissue endothelin-1 levels in chronic liver disease correlate with disease severity and ascites. Am J Gastroenterol. 2000;95:199-203. doi: 10.1111/j.1572-0241.2000.01684.x.

28. Lin CY, Tsai IF, Ho YP, et al. Endotoxemia contributes to the immune paralysis in patients with cirrhosis. $J$ Hepatol. 2007;46:816-826. doi:10.1016/j.jhep.2006.12.018.

29. Cahill PA, Redmond EM, Sitzmann JV. Endothelial dysfunction in cirrhosis and portal hypertension. Pharmacol Ther. 2001;89:273-293. doi:10.1016/S0163-7258(01)00128-0.

30. Iwakiri Y, Groszmann RJ. Vascular endothelial dysfunction in cirrhosis. J Hepatol. 2007;46:927-934. doi:10.1016/j.jhep.2007. 02.006 .

31. Bosch J. Vascular deterioration in cirrhosis: the big picture. $J$ Clin Gastroenterol. 2007;41:S247-S253.

32. Schraufnagel DE, Kay JM. Structural and pathologic changes in the lung vasculature in chronic liver disease. Clin Chest Med. 1996;17:1-15. doi:10.1016/S0272-5231(05)70295-1.

33. Shiraishi K, Matsuzaki S, Ishida $H$, Nakazawa $H$. Impaired erythrocyte deformability and membrane fluidity in alcoholic liver disease: participation in disturbed hepatic microcirculation. Alcohol Alcohol Suppl. 1993;1A:59-64.

34. Myers PR, Parker JL, Tanner MA, Adams HR. Effects of cytokines tumor necrosis factor alpha and interleukin 1 beta on endotoxin-mediated inhibition of endothelium-derived relaxing factor bioactivity and nitric oxide production in vascular endothelium. Shock. 1994;1:73-78.

35. Giron-Gonzalez JA, Martinez-Sierra C, Rodriguez-Ramos C, et al. Adhesion molecules as a prognostic marker of liver cirrhosis. Scand J Gastroenterol. 2005;40:217-224. doi:10.1080/ 00365520510011470.

36. Stenmark KR, Fagan KA, Frid MG. Hypoxia-induced pulmonary vascular remodeling: cellular and molecular mechanisms. Circ Res. 2006;99:675-691. doi:10.1161/01.RES.0000243584.45145.3f.

37. Baik SK, Jee MG, Jeong PH, et al. Relationship of hemodynamic indices and prognosis in patients with liver cirrhosis. Korean $J$ Intern Med. 2004;19:165-170. 